\title{
Multifocal nodular lymphoid hyperplasia of the lung
}

\author{
Gil Tae Lee ${ }^{1}$, Eun Kyoung Kim¹, Eirie Cho', Seung-Sook Lee ${ }^{2}$, Seo Yun Kim¹, \\ Cheol Hyeon $\mathrm{Kim}^{1}$, Hye-Ryoun $\mathrm{Kim}^{1}$ \\ Departments of ${ }^{1}$ Internal Medicinel and ${ }^{2}$ Pathology, Korea Cancer Center Hospital, Korea Institute of Radiological and \\ Medical Sciences, Seoul, Korea
}

\begin{abstract}
Nodular lymphoid hyperplasia (NLH) is a benign lymphoproliferative disease that can affect the lung. Because of its rarity, little is known about the etiology and natural history of NLH. Most cases are usually asymptomatic and found incidentally on imaging studies. Imaging finding of NLH has shown most commonly as a solitary lesion, although multifocal pulmonary nodules may be seen. Surgical resection has proved curative in the cases previously described. We report a rare case of NLH in a 55 year-old man who presented with bilateral multiple pulmonary nodules on chest radiography. Open biopsy was performed from the upper and lower lobe of the left lung. The lesions were pathologically diagnosed as pulmonary NLH. Multifocal residual nodules in both lungs remain stable without spontaneous regression during the 3 years of follow-up.
\end{abstract}

Keywords: Lung; Pseudolymphoma; Lymphoproliferative disorder

\section{INTRODUCTION}

Pulmonary nodular lymphoid hyperplasia (NLH) is a term first suggested by Kradin and Mark to describe one or more nodules or infiltrates composed of reactive lymphoid cells [1]. Most patients affected with pulmonary NLH are asymptomatic and typically discovered incidentally because of abnormal chest radiographs. In Abbondanzo's series, the most common imaging finding in patient with NLH was a solitary pulmonary nodule or mass, and about one-thirds of patients had multifocal pulmonary lesions [2]. Because curative surgeries were performed in the most cases, radiological changes of residual NLH have infrequently been reported. Herein, we present a case of pulmonary NLH with stable multifocal

Received: July 29, 2015, Revised: September 1, 2015 Accepted: September 23, 2015

Corresponding Author: Hye-Ryoun Kim, Department of Internal Medicine, Korea Cancer Center Hospital, Korea Institute of Radiological and Medical Sciences, 75 Nowon-ro, Nowon-gu, Seoul 01812, Korea Tel: +82-2-970-1206, Fax: +82-2-970-2438

E-mail: drhwpark@naver.com nodules during the 3 years of follow-up. And NLH is a very rare disease in Korea. So far, only 5 cases including our present case have been reported in the Korean literature.

\section{CASE}

A 55-year-old man had an abnormal shadow revealed in chest radiography images during a regular checkup in a local hospital. The patient was referred to our hospital. Computed tomography showed a well demarcated mass in the left upper lobe of the lung, measuring $3 \mathrm{~cm}$ in diameter. In addition, multiple small lesions showed nodular opacity and ground glass attenuation (Fig. 1). There were no contributory findings in the medical and social history except 30 years of smoking. No remarkable findings were present at physical examinations. Laboratory data were unremarkable.

A bronchofiberscopic examination was performed, but it did not lead to a diagnosis. Then, transthoracic needle aspiration biopsy was performed targeting the mass in the left upper lobe. However, the biopsy was insufficient for a diagnosis. We decided to perform a video-assisted thoracoscopic sur-

Copyright (C) 2017 Yeungnam University College of Medicine

This is an Open Access article distributed under the terms of the Creative Commons Attribution Non-Commercial License (http://creativecommons.org/licenses/by-nc/4.0/) which permits unrestricted non-commercial use, distribution, and reproduction in any medium, provided the original work is properly cited. 
gery. The patient underwent wedge resections for the upper and lower lobe of the left lung.

A total of 6 lesions, including 5 lesions in the left upper lobe (lingular segment, measuring $8.5 \times 5.2 \times 1.5 \mathrm{~cm}$ and 25.6 g). There are 5 tumorous lesions, measuring $1.3 \times 1.4 \mathrm{~cm}$, $1.1 \times 1 \mathrm{~cm}, 1.6 \times 0.8 \mathrm{~cm}, 1.1 \times 0.7 \mathrm{~cm}$, and $0.8 \times 0.7 \mathrm{~cm}$ and 1 tumorous lesion, measuring $1.4 \times 1 \mathrm{~cm}$, in the left lower lobe (posterobasal segment, measuring $6 \times 1.5 \times 1 \mathrm{~cm}$ and $4.2 \mathrm{~g}$ ). The resected lesions were well-circumscribed, tan-white-gray, round and firm nodules. These nodules are large and well demarcated. In addition some nodules are subpleural predilection. So follicular bronchiolitis were excluded. An aspiration cytology test performed during the operation showed clustered lymphoid cells, which was interpreted as suspicious for low grade lymphoma. On pathological examination, all specimens showed relatively well circumscribed mass composed of reactive follicles in lung parenchyma, reactive follicles with no involvement of lymphoid cells in adjacent bronchial epithelium. Hematoxylin-eosin (H\&E) staining did not show a marginal zone, lymphoepithelial lesions, or sheets of plasma cell proliferation. Cellularity were reactive germinal centers with interfollicular small lymphocytes and plasma cells. Germinal centers were reactive with no follicular colonization by neoplastic cells. And immunohistochemical test results were dis- tin- guished. CD3 is positive in germinal center. CD20 is positive in follicles and negative in interfollicular area. Bcl-2 is negative in germinal center and positive in mantle zone. Ki-67 is high proliferation index in germinal center (Fig. 2). B cell Immunoglobulin heavy chain gene rearrangement is no monoclonality (Fig. 3). These histological findings resulted in a diagnosis of NLH. The postsurgical course was uneventful, and no further therapy was given.

During a 3-year follow-up, multifocal residual nodules in both lungs remain stable without spontaneous regression.

\section{DISCUSSION}

$\mathrm{NLH}$ is a rare benign disease showing pulmonary indurations due to the propagation of reactive lymphocytic cells and their infiltration into the lungs, with radiological findings appearing similar to those observed in pneumonia or adenocarcinoma. Its pathological differentiation from mucous associated lymphoid tissue (MALT) lymphoma is important. Previously, this disease was named pseudolymphoma by Salzstein [1-4]. According to a report by Abbondanzo et al., this disease is mostly detected due to abnormalities on a chest radiograph while the patient is asymptomatic; however, it could also lead to symptoms such as breathing difficulties, coughing (along
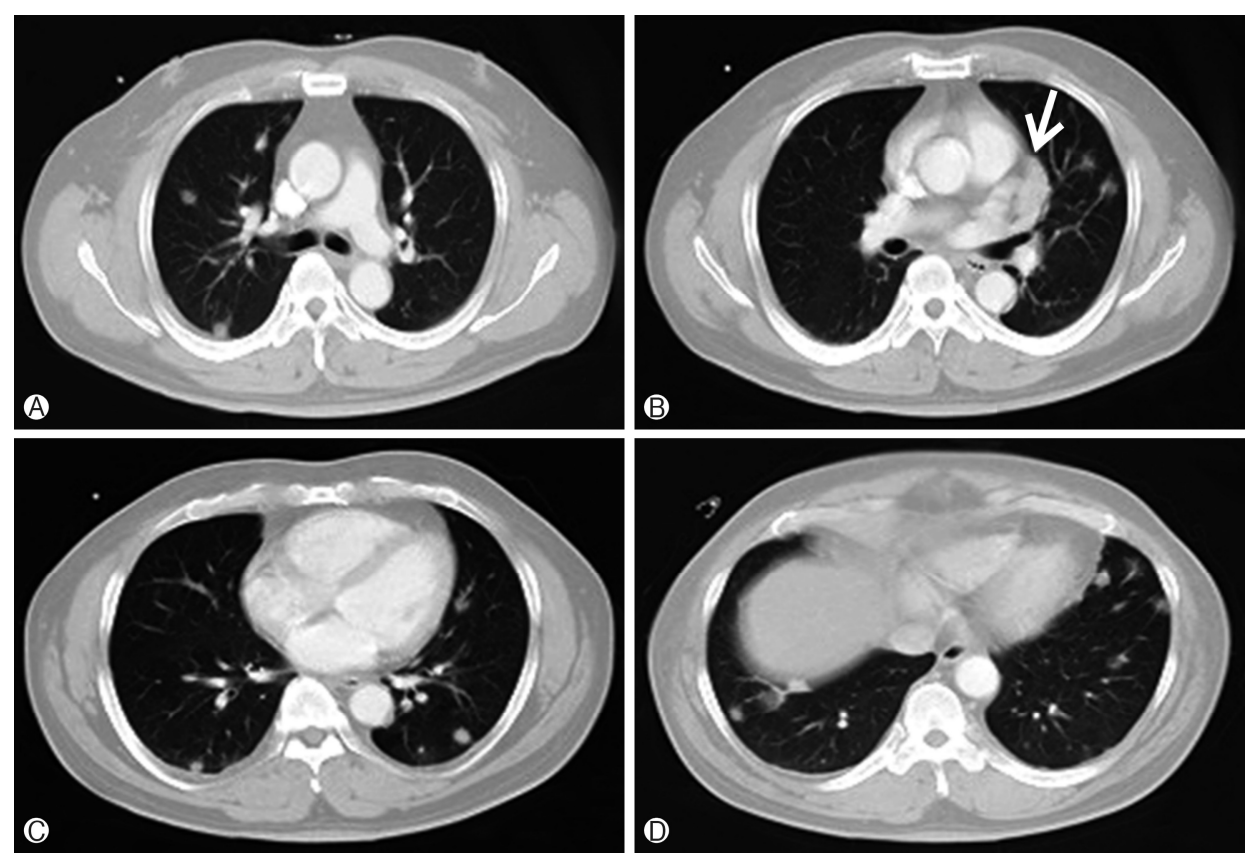

Fig. 1. 3-cm sized mass (arrow) in the left upper lobe of the lung and multiple small nodular shadows in both lungs were shown at preoperative chest computed tomography (April 10, 2012). 

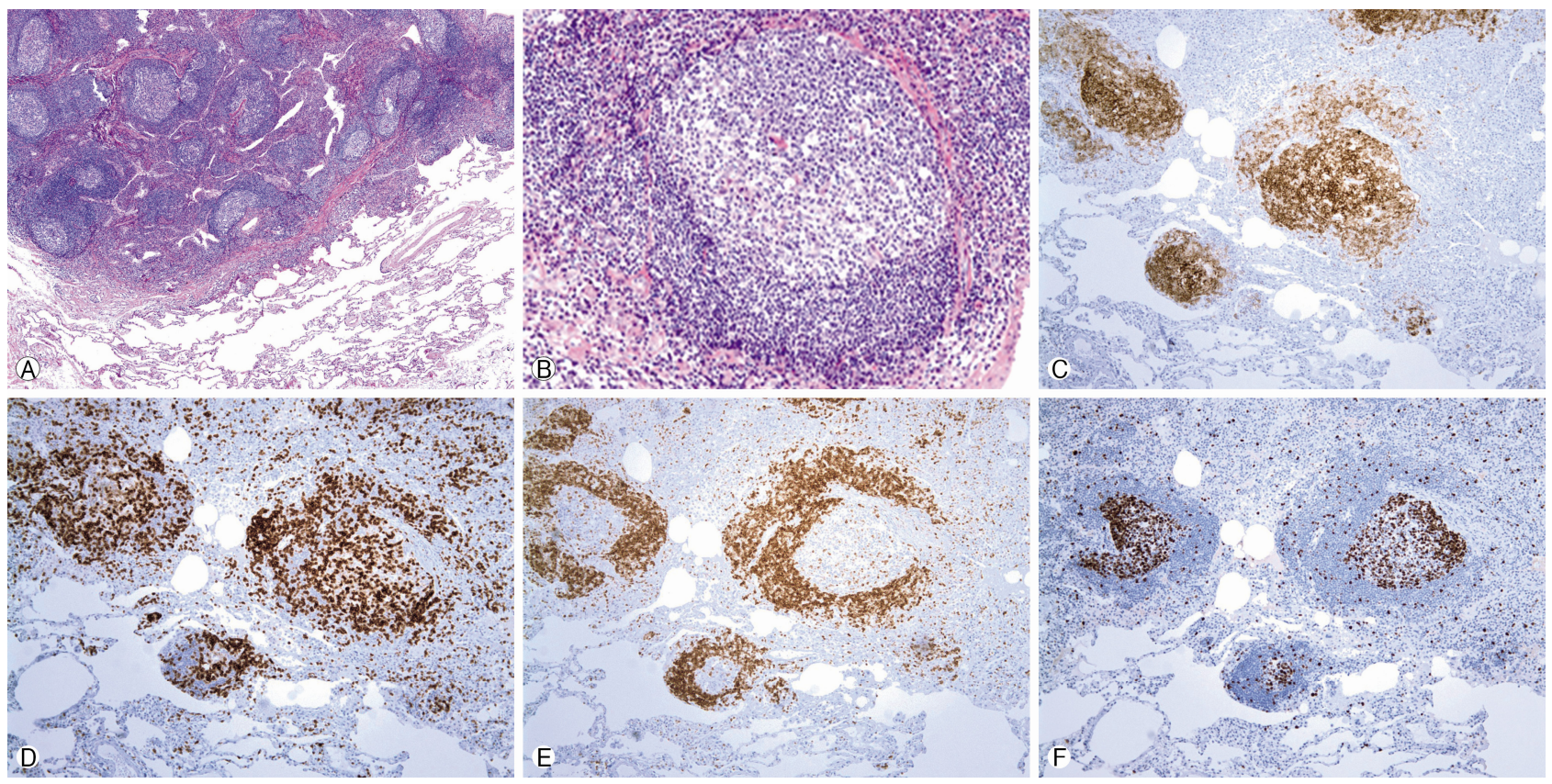

Fig. 2. (A) Reactive follicles with no involvement of lymphoid cells in adjacent bronchial epithelium (H\&E stain, $\times 40)$. (B) Reactive follicles; polarization, cellular heterogeneity, tingible body macrophage (H\&E stain, $\times 200)$. (C) CD20: positive in follicles, negative in interfollicular area. (D) CD3: positive in germinal center. (E) Bcl-2: negative in germinal center, positive in mantle zone. (F) Ki-67: high proliferation index in germinal center.

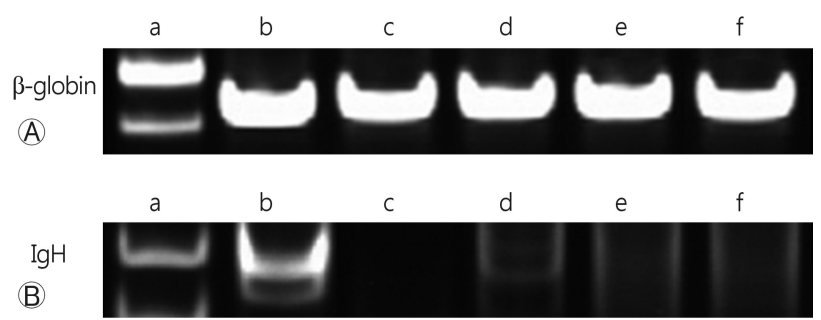

Fig. 3. (A) PCR ( $\beta$-globin). a) Size marker, b) positive control (Raji cell), c) negative control (Jurkat cell), d) patient's sample $10 \mathrm{ng}$, e) patient's sample $50 \mathrm{ng}, \mathrm{f}$ ) patient's sample $100 \mathrm{ng}$. (B) B cell immunoglobulin heavy chain gene rearrangement: no monoclonality. a) Size marker, b) positive control (Raji cell), c) negative control (Jurkat cell), d) patient's sample $10 \mathrm{ng}$, e) patient's sample $50 \mathrm{ng}$, f) patient's sample $100 \mathrm{ng}$. IgH, immunoglobuline H; PCR, polymerase chain reaction.

with production of sputum), pleuritic chest pain, and febricity. The most vulnerable ages are $50-80$ years, and there is no difference in incidence between men and women. Despite there being no known causes, it is speculated to be caused by immune stimulation; it was diagnosed in a patient with Sjögren syndrome, although the association was not verified [1-7].

NLH is characterized by a sharp border separating it from the surrounding tissue, unclear nodules, or nodular opacity on chest radiographs [2]. In most cases, it exists as a nodule with a sharp border under the pleura, and sometimes shows ground-glass opacification on computerized tomography. Although it may show single or multiple lesions, it rarely exists as multiple bilateral lesions. Radiologically, it appears similar to adenoma, bronchioloalveolar carcinoma, and MALT lymphoma. However, it is difficult to diagnose NLH only on the basis of imaging results. Hence, a surgical diagnosis is required in many cases, as a preoperative histological diagnosis is difficult to make. Since malignant diseases or interstitial lung diseases could not be excluded by radiological examination alone, a surgical diagnosis was also performed in the present case.

Pathologically, it is known that multiple plasma cells are observed in the space between the reactive germinal center and follicles in lymphoid tissues, while lymphoepithelial lesions, tabular like invasion of the pleura, and invasion of bronchial cartilage are not observed [2]. It is also known that propagation of $\mathrm{B}$ cells expressing CD20 in the germinal center, and lymphocytes in the follicles expressing CD43, CD3, and CD5 is observed on immunohistochemical tests, and that most of the immunoglobulin light chains show a polyclonal reaction, which distinguishes it from MALT lymphoma [2-5]. 
$\mathrm{Bcl}-2$ in the germinal center should not be stained, and no rearrangement of the immunoglobulin heavy chain genes should be observed on molecular genetic testing. During histological analysis, it is important to differentiate nodular lymphoid hyperplasia from lymphocytic interstitial pneumonia and MALT lymphoma [2,7].

MALT lymphoma sometimes shows bilateral multiple nodules and lymphogenous metastasis, as well as diffuse infiltrating lesion that may invade pleura and bronchial cartilage [6]. Well-fixed and well-processed H\&E and immunohistochemically stained paraffin sections are usually sufficient to differentiate nodular lymphoid hyperplasia from MALT lymphoma of the lung. However, there may be cases that are difficult to diagnose because of sparse tissue size or suboptimal fixation or processing. For such cases, ancillary techniques such as molecular genetic analysis may be helpful. $40 \%$ of the immunoglobulin light chain reactions are monoclonal reactions in MALT lymphoma, and rearrangement of the immunoglobulin heavy chain genes is observed in most cases $[2,6]$.

Lymphocytic interstitial pneumonia and follicular bronchio litis invade the entire lung diffusely, rather than generating nodules [2]. Thin-section computed tomography feature of follicular bronchiolitis consists of small centrilobular nodules variably associated with peribronchial nodules and areas of ground-glass opacity [8]. Focusing on the grossly inspection of the trachea can view the nodule with a diameter of 1-2 $\mathrm{mm}$, It can be diagnosed when the lesion has seen the proliferation of lymphoid reactive germinal center with lymphocyte infiltration around the bronchioles in the center of the optical microscopic [9].

Treatment of nodular lymphoid hyperplasia involves surgical excision. Although it has been reported that the chance of postoperative recurrence is significantly low [5], careful follow-up is required as it is hard to completely exclude MALT lymphoma. In particular, if multiple nodules on both lungs are gradually growing, nodular lymphoid hyperplasia should be suspected.
In the present case, the patient was admitted to the hospital because of abnormal radiological findings, and was diagnosed with NLH by surgical biopsy that was performed to exclude malignant diseases and interstitial lung diseases.

NLH cannot be accurately diagnosed, even by biopsy, unless extra staining is performed. As a result, there are chances that it can be misdiagnosed as MALT lymphoma, resulting in incorrect treatment. Hence, although this is a rare disease, it must not be ignored by physicians.

\section{CONFLICT OF INTEREST}

No potential conflict of interest relevant to this article was reported.

\section{REFERENCES}

1. Kradin RL, Mark EJ. Benign lymphoid disorders of the lung, with a theory regarding their development. Hum Pathol 1983; 14:857-67.

2. Abbondanzo SL, Rush W, Bijwaard KE, Koss MN. Nodular lymphoid hyperplasia of the lung: a clinicopathologic study of 14 cases. Am J Surg Pathol 2000;24:587-97.

3. Saltzstein SL. Pulmonary malignant lymphomas and pseudolymphomas: classification, therapy, and prognosis. Cancer 1963;16:928-55.

4. Nicholson AG. Pulmonary lymphoproliferative disorders. Curr Diagn Pathol 2000;6:130-9.

5. Koss MN. Pulmonary lymphoid disorders. Semin Diagn Pathol 1995; 12:158-71.

6. Li G, Hansmann ML, Zwingers T, Lennert K. Primary lymphomas of the lung: morphological, immunohistochemical and clinical features. Histopathology 1990;16:519-31.

7. Guinee DG Jr. Update on nonneoplastic pulmonary lymphopro liferative disorders and related entities. Arch Pathol Lab Med 2010;134:691-701.

8. Howling SJ, Hansell DM, Wells AU, Nicholson AG, Flint JD, Müller NL. Follicular bronchiolitis: thin-section CT and histologic findings. Radiology 1999;212:637-42.

9. Ryu JH, Myers JL, Swensen SJ. Bronchiolar disorders. Am J Respir Crit Care Med 2003;168:1277-92. 